anthropology \& materialism

\section{Anthropology \& Materialism}

A Journal of Social Research

$4 \mid 2019$

Art and Technique: A Framework of Unaccomplished Promises

\title{
Quelques bouts de pellicule, quelques gestes politiques
}

Entretien avec Georges Didi-Huberman, par Ilana Feldman. Avec une introduction d'Ilana Feldman

Ilana Feldman et Georges Didi-Huberman

(2) OpenEdition

Journals

Édition électronique

URL : http://journals.openedition.org/am/1485

DOI : 10.4000/am. 1485

ISSN : $2364-0480$

Éditeur :

CETCOPRA, CRASSH - Center for Research in the Arts Social Sciences and Humanities, Fakultät Gestaltung - Universität der Künste Berlin

Référence électronique

Ilana Feldman et Georges Didi-Huberman, «Quelques bouts de pellicule, quelques gestes politiques », Anthropology \& Materialism [En ligne], 4 | 2019, mis en ligne le 22 juin 2019, consulté le 23 novembre 2019. URL : http://journals.openedition.org/am/1485; DOI : 10.4000/am.1485

Ce document a été généré automatiquement le 23 novembre 2019.

Tous droits réservés 


\section{Quelques bouts de pellicule, quelques gestes politiques}

Entretien avec Georges Didi-Huberman, par Ilana Feldman. Avec une introduction d'Ilana Feldman

Ilana Feldman et Georges Didi-Huberman

\section{NOTE DE L'AUTEUR}

Le texte qui suit fut publié comme supplément à Ecorces, ouvrage publié au Brésil par Editora 34 en 2017. Dans cet entretien qui nous fut accordé en septembre de la même année par e-mail, Georges Didi-Huberman a parfois réuni plusieurs questions afin de développer ses réponses plus librement.

Le contexte est le suivant : en juin 2011, le philosophe et historien de l'art se rend à Auschwitz-Birkenau, son appareil-photo à la main, à la manière d'un visiteur lambda du moins jusqu'à un certain point. Huit ans ont passé depuis la publication de son ouvrage Images malgré tout (2004), où il analysait quatre images prises clandestinement dans la zone du crématoire $n^{\circ} 5$ de Birkenau, en août 1944, par un membre du Sonderkommando, ce groupe de prisonniers juifs chargés d'en conduire d'autres jusqu'aux chambres à gaz, puis de transporter les corps jusqu'aux fours crématoires. L'auteur décide de revisiter son enquête en « revenant au lieu » : lieu de mémoire, mais aussi de débats voire d'intenses polémiques.

Petit-fils de juifs polonais assassinés ici-même, dans les chambres à gaz du plus grand camp d'extermination du Troisième Reich, Didi-Huberman commence sa déambulation en recueillant trois morceaux d'écorce, surface tout aussi fragile que les pellicules photographiques qui nous sont parvenues par un photographe clandestin, et qui sont les seuls témoignages visuels que nous ayons du génocide. Face à cette fragilité, face aux vestiges, à ce qui reste dans l'espace désolé du camp, face à sa propre émotion aussi, il se demande : «Une fois que je serai mort, que pensera mon fils lorsqu'il tombera sur de tels résidus?» 
Mélange d'essai, de narration photographique et de récit d'expérience, texte en même temps poétique et philosophique, doté d'un style clair et dense, Ecorces peut aussi être lu comme une lettre. Une lettre aux générations futures, qui vise à interroger les modes de construction de la mémoire, les possibilités de transmission d'une connaissance sensible, mais qui vise par-dessus tout à questionner notre propre regard. Dans Ecorces, Didi-Huberman nous rend plus évident que jamais le fait que l'image n'est pas une icône, une représentation, un document ou la preuve d'une vérité, mais un acte collectif, un geste qui ne cesse d'être politique, enraciné dans un véritable travail du regard.

A travers un montage de fragments, Didi-Huberman observe tout ce qui l'environne comme un archéologue, déterrant le passé et comparant ce que nous voyons au présent, ce qui a survécu, avec ce que nous savons qui a disparu. Mais cela n'arrive pas sans douleur. Il n'est pas rare que l'historien des images se mette en scène, notant discrètement la sensation douloureuse qu'une telle traversée provoque en lui, exprimée dans sa manière d'aller, la tête plus basse que d'habitude, le regard au ras des choses. Il ne s'agit cependant pas ici de faire de la douleur un privilège, une réserve d'exclusivité, comportement fréquent dans les discours de victimisation. Bien au contraire : comme Didi-Huberman l'affirme dans l'entretien qui suit, il convient de faire de la douleur et des émotions qui nous accompagnent un bien commun, partageable et transmissible. Car c'est seulement par la transmission que nous devenons capables de ne pas nous résigner devant les impasses de l'entendement. Que nous pouvons, pardessus tout, penser, dire, voir, réfléchir et, surtout, imaginer. Ce n'est pas pour une autre raison que, devant l'« inimaginable », l'imagination s'affirme comme une nécessité politique.

Ilana Feldman : Écorces est un livre singulier dans votre trajectoire. Mélange d'essai, de récit de voyage et de narration biographique, texte à la fois poétique et philosophique, au style clair et dense, on peut aussi le lire comme une lettre. Une lettre pour les générations futures, à commencer par votre fils. Dès le début, alors que vous contemplez les trois morceaux d'écorces que vous avez recueillies lors d'un voyage à Auschwitz-Birkenau, vous vous demandez : "Que pensera mon enfant lorsqu'il tombera, moi mort, sur ces résidus?» De tous vos essais, Écorces ne serait-il pas celui qui, partant de votre propre finitude et problématisant les modes de construction de la mémoire, serait le plus concerné par la question de la transmission?

L'ancrage autobiographique du texte, peu fréquent dans votre œuvre, attire nécessairement l'attention. II est frappant de vous voir tracer, à travers un travail de montage de fragments, un fil qui va du désir de futur (votre enfant, les générations suivantes) à un passé ébréché, en miettes (vos grands-parents, entre des millions d'autres, assassinés ici même, à Auschwitz), dont il faut faire la fouille. Au cours de ce cheminement, vous vous mettez vous-même en scène, pointant discrètement la «sensation pénible ", le «serrement de cœur » et l'« accablement particulier » que cette traversée produit en vous et qui s'exprime par une manière de marcher la tête plus basse que d'habitude, le regard vers «les choses terre à terre ». Pourtant, quelques années après avoir écrit Écorces, vous commencez votre livre Peuples en larmes, peuples en armes (Minuit, 2015) avec l'exergue suivante, de Gilles Deleuze: «L'émotion ne dit pas “je". [...] On est hors de soi. » Que pensez-vous des écritures de soi et de l'inflation de l'autobiographique aujourd'hui ? Y aurait-il quelque recours, une juste distance, pour l'expression du «je »?

Georges Didi-Huberman : Chaque livre est singulier, sans doute. L'auteur aurait même tendance, en général, à exagérer la singularité de chacun de ses textes. En sorte que la véritable singularité lui échappe en grande partie. Écorces, en ce sens, répète autant qu'il innove dans mon cheminement d'écriture. Le fait qu'il soit un livre bref n'est 
pas, en soi, une singularité : il m'est depuis longtemps nécessaire de faire varier les formats comme les rythmes de mes livres. Il y a, d'un côté, de longues recherches à épisodes qui supposent une opiniâtreté et une patience de longue durée, comme dans les séries d'ouvrages sur le thème de la nymphe, sur l' « œil de l'histoire » en six volumes, ou encore sur les soulèvements, qui est un projet en cours. D'un autre côté, l'impatience d'un texte bref apparaît souvent comme nécessaire, libératoire, comme lorsque vous prenez, depuis la longue route de vos "projets », un chemin de traverse inattendu qui est l'indicateur plus immédiat de vos "désirs", fussent-ils inconscients. Se donner, s'offrir le temps de soudain bifurquer, d'abandonner provisoirement toute idée de "projet ", voilà un aspect essentiel, à mon sens, de la liberté d'écrire et de penser.

Vous soulignez l'«ancrage autobiographique » de ce petit texte, et vous dites que c'est « peu fréquent » chez moi... Vous avez raison sur un plan de lecture explicite c'est, en effet, un texte où le « je » est assumé comme tel - mais, en réalité, chaque parcelle de mon travail est mû, que ce soit selon un détour ou pour une raison directe, par un motif d'expérience ou un « ancrage autobiographique ». Et cela pour la simple raison qu'en choisissant un domaine de recherche on se confronte à quelque chose qui, dans la vie intime, nous a fatalement touché. Ce fut le cas, par exemple, pour mon premier livre sur la Salpêtrière : l'ancrage était là, évident à mes yeux - mais camouflé à ceux du lecteur -, et c'était celui d'une expérience précédemment vécue dans l'espace hospitalier. Il y a cependant, dans Écorces, deux dimensions autobiographiques plus « singulières » en effet : c'est la place du je et c'est celle du juif. Le je est assumé en tant que ce texte est le récit d'une expérience. Cela au triple sens des mots allemands Erfahrung ou Erlebnis, "expérience vécue»; Experiment, "expérimentation"; et, enfin, Erkenntnis, "expérience acquise, connaissance »...

La place du juif est plus problématique. Jusqu'à Écorces, je ne crois pas avoir jamais assumé ma position intellectuelle et publique à partir de ma condition de juif. C'est même l'un des bonheurs de la condition laïque du débat intellectuel en France : je n'avais pas eu à décliner la religion de mes pères - comme on dit en français, mais pour le judaïsme il vaudrait mieux dire : "la religion de mes mères » - pour avoir toute légitimité à parler sur la théologie négative chez le Pseudo-Denys l'Aréopagite ou sur l'iconographie christique chez Fra Angelico. La place du juif m'a été assignée polémiquement et négativement - en tant que «mauvais juif » ou transfuge - par d'autres «juifs publics", Claude Lanzmann et Gérard Wajcman, dans le cadre des violents débats qui ont eu lieu, en 2001, autour de Images malgré tout. Bref, je ne me suis jamais désigné moi-même, dans la sphère publique et intellectuelle, comme juif : ce sont plutôt d'autres juifs qui m'ont accusé d'être une sorte de renégat. De la sorte je suis devenu, malgré moi, un « juif non juif » aux yeux de certains, un « juif public » de toute façon. Cela m'obligeait, en quelque sorte, à me situer dans le judaïsme français, ce qui n'a rien d'aisé et continuera sans doute de me faire prendre position, ici ou là.

C'est une position difficile à construire. Notamment à cause de la référence que vous faites à cette magnifique phrase de Deleuze : «L'émotion ne dit pas je... »Car, lorsque vous arpentez le site de Birkenau, il est évident que la dimension du juif et celle du je se rencontrent dans quelque chose qui est, bien sûr, une émotion. La position est difficile à construire parce qu'elle ne doit rien affirmer sous l'angle d'une "élection » 
quelconque : cela me semble constituer la base même d'une éthique de l'écrivain visà-vis de son lecteur. Personne n'est « élu » pour quelque destin que ce soit. On n'est donc pas « élu » parce qu'on a un diplôme de philosophie, parce qu'on écrit, parce qu'on est juif ou pour toute autre raison. C'est à nous, et non à quelque instance supérieure ou abstraite, d'élire, c'est-à-dire de désigner, de choisir, d'aimer. Donc : ni juif comme peuple élu, ni je comme sujet supérieur. La difficulté : assumer tout de même une subjectivité et une histoire sans mettre son propre moi ou sa propre généalogie au centre de tout. La voie pour y parvenir : ouvrir les yeux sur les autres, sur le monde alentour, empiriquement, attentivement, modestement. Faire confiance à l'imagination. Dialectiser son regard. Regarder les barbelés d'Auschwitz avec les ramures des arbres, les cheminées avec les eaux dormantes. Puis écrire comment tout cela peut être regardé ensemble.

I.F. : Écorces est traversé - et il ne pouvait peut-être pas en être autrement avec un tel sujet - par une douleur, par une émotion, une manière d'être affecté par la surface même de ce qui reste. Lors d'une conférence destiné aux jeunes d'une dizaine d'années intitulée Quelle émotion! Quelle émotion?, vous soutenez que, au contraire d'une certaine tradition philosophique qui a toujours privilégié le domaine de la raison, le logos, c'est le domaine de l'émotion, le pathos, qui met le corps en mouvement, formant une ouverture effective en direction d'un type de connaissance sensible et de transformation active de notre monde. De quelle manière cette perspective fut-elle décisive pour votre méthode de travail avec les images et dans le cadre de l'histoire de l'art? Et, dans votre vie, serait-ce cette douleur qu'on pourrait peut-être nommer une douleur fondamentalement juive - qui vous stimule à vous confronter aux impasses de la pensée et aux limites de notre capacité d'entendement?

«Pour savoir il faut s'imaginer», répétez-vous avec insistance alors que vous cherchez à comprendre les conditions de la production de quatre images prises clandestinement, dans le danger et la précarité, par un membre du Sonderkommando au Crématoire $V$ de Auschwitz-Birkenau en août 1944. En accord avec la thèse que vous développez dans Images malgré tout (Minuit, 2003) et reprise dans Écorces, ces quatre photographies, seuls testaments visuels du génocide produits par les prisonniers eux-mêmes, aussi lacunaires, partiaux et incomplets soient-ils, "s'adressent à l'inimaginable et le réfutent ». Devant la rampe de tri de Birkenau, ou les «non-aptes» (surtout les femmes, les enfants et les personnes âgées) étaient envoyés directement dans les chambres à gaz, vous auriez vousmême dit, comme vous le relatez dans Écorces: "C'est inimaginable». Pour ajouter aussitôt: " donc je dois l'imaginer malgré tout ». Qu'y a-t-il d'extrêmement problématique voire même d'ambivalent dans la présentation, par de nombreux artistes et théoriciens, de Auschwitz comme un événement « inimaginable », « indicible » et « impensable » ?

G. D-H. : «Une douleur fondamentalement juive », dites-vous. Oui... Oui et non. Oui, cela est vrai subjectivement et généalogiquement pour ce qui concerne mon histoire familiale. Mais il me faut aussi vous répondre: non. Non, parce qu'une histoire familiale - voire une histoire élargie au domaine d'un groupe religieux, par exemple - ne dit jamais, ni le dernier mot de l'histoire, ni le dernier mot de la douleur. On ne possède pas la douleur, c'est elle qui nous possède. De plus je suis chercheur et non pas militant d'une cause où se résumerait toute mon identité ; c'est pourquoi j'ai pu travailler, à l'occasion, sur les camps d'internement des Républicains espagnols en France ou sur le sort fait aux réfugiés dans l'Europe d'aujourd'hui. Ce qu'on appelle « victimisation », « devoir de mémoire », et qui fait l'objet de tant d'abus, consiste à faire de la douleur un dû, un mot d'ordre, un capital psychique, un fonds d'investissement politique ou que sais-je encore. C'est souvent, d'ailleurs, une façon de dévaloriser la douleur des autres. Or la douleur ne se quantifie pas. La douleur ne s'échange pas contre quoi que ce soit d'autre. C'est en cela qu'elle est « inestimable », 
c'est-à-dire, en un sens, sacrée. Quand je visite Birkenau, la douleur de ceux qui sont morts ici et, par conséquent, mon émotion présente, sont "fondamentalement juives", sans aucun doute. Mais rien ne m'autorise à croire que j'aurais un quelconque droit ou privilège sur cette douleur-là, même si mes grands-parents sont morts à Birkenau. Me considérer comme propriétaire de cette douleur serait abject et méprisant pour toutes les autres douleurs du monde. Croire posséder ce dont j'ai psychiquement hérité serait ignoble: une attitude de parvenu, comme eût dit Hannah Arendt. M'identifier à cette douleur serait tout autant abusif, erroné, narcissique.

Alors, quoi ? Eh bien il suffit - mais c'est tout un travail - de faire de la douleur et, donc, de l'histoire et des émotions qui vont avec, nos biens communs : nos objets de pensée à échanger, et non pas nos chasses gardées. Inversement, il faut se méfier de la méfiance systématique dont l'émotion est l'objet chez de très nombreux intellectuels en Occident. Lorsque je vois Hal Foster, un critique d'art américain fort respectable au demeurant, affirmer, dans la revue Artforum International: "Quand j'entends le mot affect, je sors mon Taser " (When I hear the word affect, I reach for my Taser)... je suis tout simplement consterné. Il pense, en une leçon mal digérée des Mythologies de Roland Barthes, que l'émotion ou l'affect empêcheraient toute pensée critique. Il pense qu'un affect n'est que de l'idéologie gesticulée. Il croit sans doute avec une parodie de Goebbels, en plus! - exprimer un point de vue brechtien : distanciation vs émotion. Or c'est une erreur philosophique profonde - le symptôme d'un point de vue étroitement rationaliste et moralisateur - que d'opposer le pathos au logos d'une part, à la praxis d'autre part. Sans même parler de Nietzsche et de Freud, on peut rappeler que Brecht n'a jamais rien dit contre l'émotion (qu'il ne faut justement pas confondre avec l'identification, l'objet réel de sa critique à travers la notion de distanciation).

Vous avez bien raison de parler de "connaissance sensible", et de perpétuelle "transformation active », si ce n'est du monde lui-même, en tout cas de notre regard et de notre pensée. C'est tout l'enjeu d'une approche philosophique qui refuse de séparer sans restes le monde sensible d'une part - considéré dans la tradition platonicienne, encore très vivace aujourd'hui, comme illégitime, marqué par l'illusion et la pure méconnaissance - et le monde intelligible d'autre part. C'est pourquoi, en toute simplicité aristotélicienne, j'ai pu commencer Images malgré tout avec la proposition "Pour savoir, il faut s'imaginer" (je rappelle, puisque je m'exprime en français et que ces mots doivent être traduits dans une langue que je ne pratique pas, que l'on dit « je m'imagine quelque chose » comme un équivalent de "j'imagine quelque chose", sauf que la langue a le mérite, dans l'expression $s$ ' imaginer, d'inclure le sujet parlant et imaginant dans son opération même de connaissance sensible).

L'image est un point sensible exemplaire de l'histoire, de la pensée, de la connaissance, voire de l'action politique. L'image, c'est là où tout est possible, le pire comme le meilleur, et à travers quoi il faut passer à un moment ou à un autre. En découvrant un espace de douleur tel que Birkenau, il m'a bien fallu dire, spontanément, ce que tant d'autres avant moi avaient pu dire : «C'est inimaginable ». L'inimaginable correspond ici à l'expérience vécue d'une rencontre avec un tel espace, démesuré, de douleur. Ce que j'ai critiqué philosophiquement, c'est que l'inimaginable soit devenu un dogme pour l'expérience conçue. Graver dans le marbre que la Shoah est inimaginable, c'est 
d'une certaine façon accéder au vœu même des organisateurs de la "Solution finale " qui la voulaient, en effet, inimaginable, impensable et invisible aux yeux du monde alentour (malheureusement, ça a très bien marché en dépit des informations accablantes qui circulaient depuis la Pologne).

Mais je dis bien : pour le pire comme le meilleur...

I.F.: L'un des moments les plus forts de Écorces est celui où vous faites une critique tranchante d'Auschwitz comme musée d'Etat et «lieu de mémoire». A partir de divers exemples tirés des décisions «muséographiques » de l'institution, vous montrez comment Auschwitz comme Lager, «lieu de barbarie », a été transformé en « lieu de culture » et vous vous demandez, perplexe, devant les bâtiments d'un camp d'extermination transformés en «pavillons nationaux » à la manière d'une biennale : "Mais que dire quand Auschwitz doit être oublié dans son lieu même pour se constituer comme un lieu fictif destiné à se souvenir d'Auschwitz? » Sachant que le projet nazi était de faire disparaître toutes les archives, c'est-à-dire de faire disparaître la propre disparition, cette critique est au fond la formulation d'un terrible paradoxe. Si, à l'apogée du triomphe du spectacle, on attend, selon le diagnostic du critique Jean-Louis Comolli, un spectacle qui cesse de simuler, il semble en revanche que, pour supporter un réel traumatique, nous avons à tout prix besoin d'un simulacre. Comment penser, d'après vous, une éducation après Auschwitz qui n'incline pas à simplifier, manipuler et édulcorer pour « mieux » transmettre ? Pensez-vous que le musée d'Auschwitz est un cas unique de pédagogie ambiguë, ou voyez-vous le même genre de problèmes dans d'autres « lieux de mémoire » dédiés aux victimes de la violence d'Etat?

G. D-H. : Non, je ne crois pas du tout qu'Auschwitz soit un cas unique. Ce que vous appelez "pédagogie ambiguë" se retrouve partout ailleurs. Je me souviens, par exemple, que peu de temps après la réunification de l'Allemagne, un groupe de juifs américains avaient constitué un mécénat pour la restauration de la synagogue de l'Oranienburger Strasse à Berlin : en quelques mois la coupole était devenue flambant neuve, toute dorée, alors que tout le reste de la rue ne présentait encore que les façades noircies des immeubles incendiés durant la prise de Berlin par l'Armée rouge. Cette louable entreprise de mémoire devenait donc arrogance pure et, plus encore, la meilleure incitation possible à l'antisémitisme. Je me souviens aussi que, lors d'un séjour à Mexico en 2007, on m'a fait part d'un projet de musée pédagogique où le spectateur devait être confronté dès le départ avec un "authentique wagon d'Auschwitz » - vous imaginez ? acheter un wagon à bestiaux en Pologne et le faire venir jusqu'à Mexico ? - et où, par contraste, une place minuscule était prévue, à la fin du parcours, aux drames mexicains eux-mêmes... C'est alors que la Shoah devient un alibi, un paravent, une caution plus ou moins honnêtement brandie. Par ailleurs j'ai connu les deux versions du mémorial de Yad Vashem à Jérusalem, où la constitution - évidemment cruciale et nécessaire - d'une pédagogie de la Shoah ne cesse de recroiser les enjeux politiques de la mythologie nationaliste israélienne, par exemple dans la relation établie, que Marek Edelman a hautement contesté, entre le suicide collectif de Massada, l'insurrection du ghetto de Varsovie et la fondation de l'État d'Israël.

Par différence, j'ai visité de très rigoureux " lieux de mémoire » tels que le sous-sol du Mémorial des juifs assassinés de Berlin, dont je parle brièvement dans Écorces, je crois, ou bien le site du camp de Buchenwald dont le directeur est à la fois historien et psychanalyste, ce qui apporte beaucoup à l'élaboration d'une problématique de la mémoire publique. Car le problème est bien celui-là: les pédagogies sont "ambiguës» quant elles se fixent exclusivement sur leur objet - la Shoah, par exemple - et le transforment en quelque chose comme un fétiche, demeurant 
incapables, alors, d'élaborer une certaine attitude plus mobile et plus problématique devant l'histoire. La pédagogie de l'histoire, c'est avant toute chose comprendre qu'une chose est passée et cependant ne passe pas (c'est-à-dire continue d'être coincée dans nos gorges et d'agir dans nos esprits). C'est apprendre à savoir ce que c'est que le passé, comment cela s'est passé et en quoi cela est passé en nous et y reste coincé. Il faut pour cela apprendre à regarder les vestiges, à lire les archives, à creuser le sol du temps. Il faut apprendre ce que c'est qu'un fragment de pellicule $6 \times 6$ en noir et blanc, plutôt que croire faciliter l'accès à l'histoire en colorisant à tout va pour « rendre plus vivant ».

Le rapport à établir entre culture et barbarie passe fatalement par une telle politique de la mémoire, qui ne peut être en même temps - si l'on a un peu lu Freud - qu'une politique du désir, c'est-à-dire de nos horizons d'attente ou d'espoir. Vous dites que «le projet nazi était de faire disparaître toutes les archives ", mais vous connaissez aussi ce projet nazi de faire du ghetto de Prague un musée ethnographique du peuple juif une fois qu'il n'existerait plus... Toute histoire travaille toujours sur deux tableaux en même temps. En ce qui concerne la problématique contemporaine que vous abordez à travers votre citation de Comolli [sur comment supporter un reel traumatique], je ne m'exprimerais pas, quant à moi, en terme de «simulacre » qui me semble trop négativement connoté. Je dirais simplement que l'objet d'une poétique [des images] est à peu près le même que celui d'une pégagogie (les deux étaient d'ailleurs très clairement associés par Brecht, Benjamin ou Eisenstein). Cela ne veut pas dire que les œuvres d'art doivent nous faire la leçon, bien sûr. Cela veut dire qu'une image, dans tous les cas, devrait comme tout texte savoir déchirer le cliché déjà formé par la fétichisation de la mémoire. Ce qu'il faut à chaque fois, c'est relancer les dés et poser de nouvelles questions.

I. F. : Depuis l'intense polémique qui a eu pour résultat la publication de Images malgré tout en 2003 et, plus tard, celle de Écorces en 2011, vous et Claude Lanzmann avez fini par tomber d'accord au moment de la sortie du film Le fils de Saul en 2015, récompensé d'une Palme d'Or à Cannes et d'un Oscar du Meilleur Film Etranger. Dans ce premier longmétrage de fiction, le cinéaste hongrois László Nemes met en scène, pour la première fois dans l'histoire du cinéma, l'épisode des quatre photographies prises par un membre du Sonderkommando d'Auschwitz-Birkenau en août 1944, ainsi que celui du soulèvement d'octobre de la même année, lorsque 450 résistants, alors liés à la résistance polonaise, furent massacrés. Dans votre lettre au réalisateur publiée sous le titre de Sortir du noir (Minuit, 2015), vous écrivez que Nemes retire cet août 1944 de l'obscurité et de la plus pure négativité et abstraction, c'est-à-dire du «trou noir » qui gouverne ladite impossibilité de représentation de la Shoah. Dans le film, que vous définissez aussi comme un "conte allégorique», Saul prend pour fils un garçon assassiné et cherche désespérément à lui donner des funérailles au milieu du quotidien insensé de l'extermination. II me semble que c'est la reconnaissance du fils (symbole de continuité et transcendance, même mort) et le désir de l'enterrer (le sauvant de l'annulation extrême) qui permet à Saul, lors d'un sursaut d'imagination, de se réinscrire dans l'histoire pour, d'une certaine manière, "survivre ». Certaines critiques ont cependant lu le film sous l'angle d'une «mythification » (à cause du parallèle avec Antigone) ou d'une "solution individuelle », en arguant notamment du fait que le délire de Saul mettait en péril la préparation collective du soulèvement d'octobre 1944. Comment liriez-vous le film dans cette tension entre le psychique et le politique, entre le geste individuel et les actes collectifs?

G. D-H. : « Vous avez fini par tomber d'accord... » Ce n'est pas un peu vite dit, non ? D'accord... mais sur quoi exactement? Je ne saurais pas vous le dire. Claude Lanzmann a murmuré un jugement bienveillant sur le film de Nemes, et c'est tant 
mieux. J'ai tenté, pour ma part, d'écrire simplement les quelques impressions que ce film avait fait lever en moi, tout en évitant l'attente médiatique qui voulait faire de ce film l'occasion d'une nouvelle polémique, genre «images" (de Nemes, en couleur, floues, terriblement animées, etc.) «malgré tout» (malgré l'irreprésentabilité du sujet). Je ne répondrai pas vraiment à votre question car il faudrait, pour ce faire, introduire deux nouvelles pièces dans ce dossier : d'une part le livre d'Alain Fleischer, Retour au noir, qui entend réactiver la polémique ; d'autre part ma réponse à ce livre que j'ai adressée à Alain - un ami de trente ans - mais que je ne désire pas publier isolément. Ce qui m'a frappé, dans ce nouvel épisode polémique, c'est que beaucoup de ses aspects reprennent, de façon sans doute inconsciente, les motifs de la précédente : volonté de dire ce qui est permis et ce qui interdit, de distinguer ce qui est « juif » de ce qui est « faussement juif », hantise de l'image-écran, etc.

Pour revenir à ce que vous dites sur le film lui-même, je suis bien d'accord avec l'idée qu'il y a chez le personnage de Saul un "sursaut d'imagination» qui a fondamentalement à voir avec la question de la survivance. Vous savez aussi que cette notion est complexe et, notamment, qu'elle doit se distinguer de la survie en tant que telle. Le geste de Saul est un geste de survivance (Nachleben, after-life) et non pas un geste de survie (Überleben, survival). L'aspect individuel de ce geste n'en est pas moins dirigé vers l'horizon d'une communauté, mais celle-ci - au contraire de ce qui se passe dans le goupe des Résistants - n'est pas pensée comme vivante ou agissante : elle est tout entière générée par un espace du deuil, de la mémoire des morts. Mais l'histoire racontée dans ce film est tellement invraisemblable, en général comme dans chacun de ses détails, que je n'y ai pu voir, quant à moi, qu'une parabole hassidique, une fiction allégorique voire une exégèse biblique (sur la question du fils réel et $\mathrm{du}$ «fils » héritier de Saül telle qu'elle est racontée dans le premier Livre de Samuel, XVI-XXXI).

I. F. : Images malgré tout me paraît un livre fondamental dans votre œuvre, mais aussi un tournant en ce qu'il fait émerger des questions que vous aviez auparavant travaillé (comme le concept de "survivance des images ») et des questions qui étaient encore à venir (comme l'idée du « soulèvement »). Dans ce sens, l'analyse phénoménologique des quatre photographies opère un passage entre ce qui serait, d'un côté, de l'ordre d'une « inscription survivante » et, de l'autre, d'un travail de « résistance » et de "soulèvement ». Dans Écorces, vous écrivez qu'«en se postant dans la chambre à gaz, là même où les SS le contraignaient, jour après jour, de décharger les cadavres des victimes à peine assassinées, il [le photographe clandestin] a transformé, pour quelques rares secondes volées à l'attention de ses gardiens, le travail asservi, son travail d'esclave de l'enfer, en un véritable travail résistant». Dans ces conditions - demandez-vous - son acte de témoignage ne devrait-il pas être compris comme un minuscule déplacement « de son travail de mort en travail de regard»? En prenant tout cela en compte, de quelle manière ces quatre photographies clandestines et survivantes - simplifiées et recadrées par le Musée de Auschwitz-Birkenau, refusées par Claude Lanzmann dans son documentaire Shoah, remises en scène par László Nemes dans Le fils de Saul et problématisées par vous-même dans Images malgré tout et Écorces - peuvent-elles être considérées comme une sorte de genèse de votre travail de commissaire de l'exposition Soulèvements, ouverte au SESC Pinheiros, à São Paulo, du 18 octobre 2017 au 28 janvier 2018 ?

G. D-H. : Oui, un tournant... sans aucun doute. Et, même, plusieurs tournants. D'abord, chaque juif croit spontanément tout savoir de la Shoah, comme s'il la portait « toute » en lui. C'est une illusion, bien sûr. Affronter ce sujet avec quelque précision vous bouleverse à nouveau et vous change pour toujours. Ensuite, ce fut un tournant sur le plan de cette polémique qui m'a, au début tout au moins, complètement surpris, 
déstabilisé et même effondré. Mais cette épreuve n'était que la conséquence d'un mouvement que j'avais moi-même enclenché: on pourrait dire que, dans cette querelle, "c'est moi qui ai commencé » comme disent les enfants qui se chamaillent dans la cour de récréation. C'est moi qui ai commencé de mettre en question le dogme si partagé de l'inimaginable. Je n'ai donc pas subi une violence gratuite, j'ai simplement reçu la violence d'une réaction qui était, sans doute, à la mesure de mon propre geste "sacrilège ». Cela m'a appris une chose fondamentale, ou plutôt cela a clarifié quelque chose que je ne savais qu'intuitivement : à savoir que la façon dont vous regardez, dont vous décrivez et dont vous comprenez une image est, au bout du compte, un geste politique.

Si les quatre images d'Auschwitz-Birkenau sont les vestiges "survivants" d'un certain état de la machinerie de mort nazie et, d'autre part, d'un certain état des prisonniers juifs du Sonderkommando en août 1944, cela veut dire qu'il faut réfléchir à ce que pourrait être une politique de la survivance. C'est à cette tâche que je me suis consacré après Images malgré tout comme après L'Image survivante, notamment dans un livre intitulé Survivance des lucioles, qui interrogeait la pensée de Pier Paolo Pasolini avec celle de Giorgio Agamben sur la notion d'apocalypse historique. Prendre quatre photos dans la zone du Crématoire $\mathrm{V}$ de Birkenau à un moment d'apocalypse meurtrière - soit à l'époque, démentielle, des convois de juifs hongrois -, c'était en quelque sorte allumer quatre minuscules lumières dans l'espace d'une immense nuit d'horreur. C'était envoyer quatre signaux lumineux, comme les bip-bip des signaux de détresse à la radio, vous savez ? C'était désespéré. Comme les autres, ou presque tous les autres, le photographe de Birkenau est mort quelques jours après avoir fait ses quatre images. Que nous puissions regarder, au creux de notre main, ces quatre bouts de planche-contact $6 \times 6$, cela signifie que nous sommes en face de vestiges, de petits bouts de peaux - c'est-à-dire des pellicules - qui « survivent » à la mort de celui qui les a faits.

Dans ce cas précis, il est aisé de comprendre qu'une "politique de la survivance", destinée à faire survivre un témoignage par-delà la mort du témoin, ne va pas sans une "politique de la résistance». On sait que la volonté, chez les membres du Sonderkommando, de faire survivre leurs témoignages - écrits ou visuels - par tous les moyens allait de pair avec l'organisation d'un soulèvement au sens strict: une tentative d'évasion doublée d'un projet pour dynamiter l'un des crématoires. Voilà peut-être en quoi le «tournant » d'Images malgré tout m'a conduit, irrésistiblement, à travailler sur la force psychique, sur le désir qui nous fait nous soulever depuis les aliénations les plus quotidiennes jusqu'aux tragédies historiques les plus extrêmes.

Je n'ai donc pas hésité à intégrer les quatre images de Birkenau dans l'exposition Soulèvements, et je l'ai fait - à ma connaissance, c'était la première fois dans un espace public - en respectant la modestie même de l'objet, à savoir cette minuscule planche-contact que les «musées de la mémoire " reproduisent si souvent, mais partiellement, en la recadrant complètement et en l'agrandissant jusqu'à en faire le papier peint de toute une salle d'exposition... Un jour, au Jeu de Paume de Paris, une spectatrice de l'exposition m'a demandé en quoi ces images avaient leur place dans une telle problématique du soulèvement, puisqu'elles ne montrent, en réalité, que des gens menés à une mort certaine ou des cadavres brûlant en tas... J'ai répondu que c'étaient les images elles-mêmes, en tant qu'actes et non pas seulement en tant que représentations, qui relevaient d'un tel geste de soulèvement. 
Bien sûr, la façon dont les images peuvent être considérées comme des opérateurs ou des gestes de soulèvement demeure toujours problématique. Il faudrait sans doute revenir à la façon dont certains philosophes - de Kant à Hannah Arendt ou de Walter Benjamin à Cornelius Castoriadis - ont pu envisager le rôle fondamental de l'imagination comme opérateur de conversion entre le rêve et l'éveil, le sensible et l'intelligible, l'esthétique et le politique, la contemplation et l'action, etc. Les images ne sont que des surfaces fragiles, des pellicules encore une fois, ce qui nous ramène au motif principal d'Écorces. Ce modeste texte, en effet, n'a été pour moi qu'une façon d'ailleurs totalement imprévue - de « revenir sur place », m'incitant à revisiter mon travail d'Images malgré tout dans l'espace physique de Birkenau. J'avais d'abord essayé de comprendre comment, plongé dans le réel implacable d'août 1944, les membres du Sonderkommando avaient décidé de transformer ce réel historique en possibilité de mémoire pour le futur, et cela par la médiation de quatre images et de quelques textes, c'est-à-dire des petits bouts de papier, de cellulose dans tous les cas. Soixante-sept ans plus tard, une fois Birkenau devenu un champ archéologique paisible et silencieux, je n'ai pu faire autrement que d'en passer par la médiation photographique de quelques images faites à la va-vite - sans même viser, pour beaucoup d'entre elles - et par leur description littéraire, pour donner forme, lacunairement, à ma propre émotion devant cette histoire. Dans tous les cas, un peu de cellulose agglomérée en pellicule aura fait office de médium. Et c'est justement la matière même qui fait l'écorce des bouleaux de Birkenau.

\section{AUTEURS}

\section{ILANA FELDMAN}

Post-doctorante à l'Ecole de Communication et des Arts de l'Université de São Paulo, Brésil

\section{GEORGES DIDI-HUBERMAN}

Philosophe et historien de l'art, directeur d'études à l'Ecole des Hautes Etudes en Sciences Sociales (Ehess), auteur d'une trentaine d'ouvrages, entre lesquels Images malgré tout (Paris : Minuit, 2004) et Ecorces (Paris : Minuit, 2011). 